\title{
Design and Characterization of a Nanocomposite Pressure Sensor Implemented in a Tactile Robotic System
}

\author{
Alessandro Massaro, Fabrizio Spano, Aimé Lay-Ekuakille, Paolo Cazzato, \\ Roberto Cingolani, and Athanassia Athanassiou
}

\begin{abstract}
In this paper, we present the implementation of a new class of optical pressure sensors in a robotic tactile-sensing system based on polydimethylsiloxane (PDMS). The sensor consists of a tapered optical fiber, where an optical signal goes across, embedded into a PDMS-gold nanocomposite material (GNM). By applying different pressure forces onto the PDMS-based nanocomposite, changes in the optical transmittivity of the fiber can be detected in real time due to the coupling between the GNM and the tapered fiber region. The intensity reduction of a transmitted light is correlated to the pressure force magnitude. Light intensity is converted into an electric signal by a system suitable for robotic implementation. High sensitivity using forces by applying weights of a few grams is proved. Sensitivity on the order of $5 \mathrm{~g}$ is checked. A detailed algorithm for the detection of roughness and shapes by means of a robotic finger is proposed.
\end{abstract}

Index Terms-Light coupling, nanocomposite materials, optical tactile sensors, pressure sensing, robotic implementation.

\section{INTRODUCTION}

$\mathbf{S}$ ENSORY information of the human skin for feeling materials and determining their physical properties is provided by sensors on the skin. Currently, many researchers are attempting to apply the five senses to intelligent robotic systems. In particular, many kinds of tactile sensors, combining small force sensors, have been introduced into intelligent robots. These tactile sensors, which are capable of detecting contact force, vibration, texture, and temperature, can be recognized as the next generation of an information collecting system. Future applications of implemented tactile sensors include robotics in

Manuscript received November 3, 2010; revised January 14, 2011; accepted January 17, 2011. Date of publication April 5, 2011; date of current version July 13, 2011. The Associate Editor coordinating the review process for this paper was Dr. Zheng Liu.

A. Massaro and F. Spano are with the Center for Biomolecular Nanotechnologies, Italian Institute of Technology, 73010 Leece, Italy (e-mail: alessandro.massaro@iit.it).

A. Lay-Ekuakille is with the Department of Innovation Engineering, Faculty of Engineering, University of Salento, 73100 Lecce, Italy.

P. Cazzato is with the National Nanotechnology Laboratory, Institute of Nanoscience, National Research Council, 73100 Lecce, Italy.

R. Cingolani is with the Italian Institute of Technology, 16163 Genova, Italy.

A. Athanassiou is with the Center of Biomolecular Nanotechnologies, Italian Institute of Technology, 73010 Leece, Italy, and also with the National Nanotechnology Laboratory, Institute of Nanoscience, National Research Council, 73100 Lecce, Italy.

Color versions of one or more of the figures in this paper are available online at http://ieeexplore.ieee.org.

Digital Object Identifier 10.1109/TIM.2011.2121290 medicine for minimally invasive microsurgery, military uses for dangerous and delicate tasks, and automation in industries. Some tactile and small force sensors using microelectromechanical system (MEMS) technology have been introduced. MEMS tactile-sensing work has been focused mainly on silicon-based sensors that use piezoresistive [1]-[3] or capacitive sensing [4]-[6]. These sensors have been realized with bulk and surface micromachining methods. Polymer-based devices that use piezoelectric polymer films [7]-[9] such as polyvinylidene fluoride for sensing have also been constructed, but polymeric piezoelectric materials are not the only ones used for sensing applications. There are a lot of different polymers investigated for this kind of application and oriented on MEMS technology. Although these sensors offer good spatial resolution due to the use of MEMS techniques, they still pick out problems in applications for practical systems. In particular, devices that incorporate brittle sensing elements such as siliconebased diaphragms or piezoresistors are not reliable for robotic manipulation [10], [11]. Previous efforts have been hindered by rigid substrates, fragile sensing elements, and complex wiring. Moreover, the polymeric solutions found in the literature for fabrication of pressure sensor systems [12]-[24] require complex fabrication processes and postprocessing analysis. All these drawbacks can be compensated by utilizing flexible optical fiber sensors and transducers. In addition, optical fiber sensors are immune from electromagnetic (EM) fields and can be easily multiplexed and integrated with small lightemitting diode sources, thus providing a good alternative for the implementation of robotic tactile sensors [25]. Moreover, the proposed optical fiber sensor is obtained by means of a simple fabrication process: A used nanocomposite material, which the fiber is embedded in, is achieved simply by chemical reduction that allows to obtain nano/micro gold particles in a polymeric material [i.e., a gold nanocomposite material (GNM)].

The use of elastomers such as polydimethylsiloxane (PDMS) has many advantages with respect to silicon or glass. PDMS is cheaper than silicon, is more flexible, and bonds easier to other materials than silicon or/and glass. PDMS conforms to the surface of a substrate over a large area and can adjust to surfaces that are nonplanar. PDMS is a homogenous and optically transparent material down to about $300 \mathrm{~nm}$. PDMS is waterproof and permeable to gases. The surface properties of PDMS can easily be changed by exposure of its surface in oxygen plasma. In this way, PDMS can bond to other materials that have a wide 
(a)

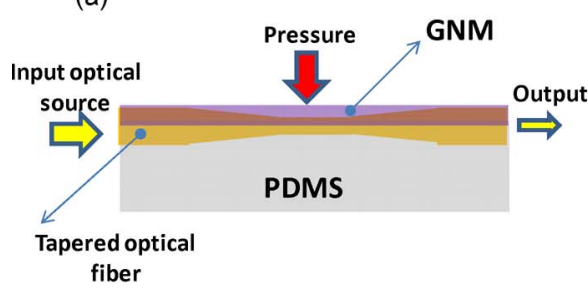

(c)

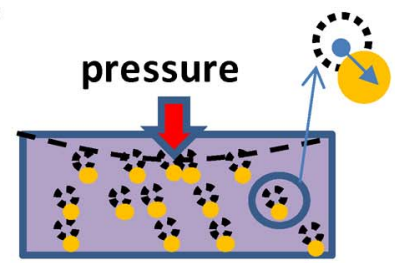

(b)

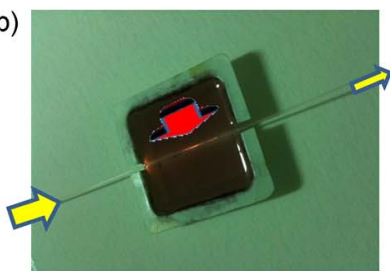

(d)

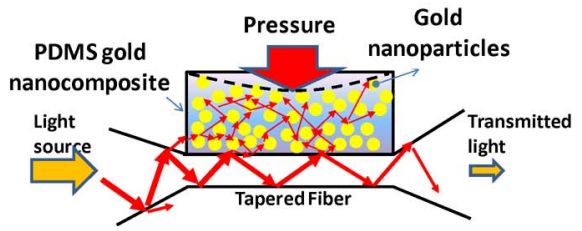

Fig. 1. (a) Schematic configuration of the optical pressure sensor prototype. (b) Photo of the fabricated prototype sensor when switched on (see illuminated fiber). (c) Displacement of gold nanoparticles in the PDMS-Au cladding due to the applied pressure force. (d) Light scattering and coupling between the tapered fiber and the PDMS-Au cladding.

range of free energy. Despite all the aforementioned advantages that the use of PDMS provides, there are some problems with PDMS.

Gravity, adhesion, and capillary forces stress the features of PDMS leading to collapse, which defects the created pattern. The adhesion between a stamp and a substrate can also cause sagging of the structures. PDMS is a shrinking material that can defect the structure of the pattern. It can also swell due to chemical reactions with some kind of nonpolar solvents such as toluene.

A PDMS polymer film was chosen for the proposed sensor due to its ability to generate gold nanoparticles starting from gold precursors [26]-[28]. Additionally, PDMS presents good elastomeric properties, which permit to obtain a realtime pressure sensor response of $0.6 \mathrm{~s}$. [13]. The use of a GNM for the detection process is simpler compared with the approaches presented in the literature [12]-[16]. The GNM supports light coupling with a tapered multimodal optical fiber and does not require complex layouts, such as membrane-type devices obtained by photolithography processes. The information of pressure detection is included in a optical transmittivity response, which decreases by applying pressure forces. Transmitting intensity can be detected and directly converted into an electrical signal by a photodiode and processed by a proper electronic circuit suitable for robotic implementation.

Therefore, in this paper, we present a newly designed optical fiber high-responsive force sensor based on an EM coupling effect.

The sensor reported is an optimized version obtained after previous preliminary studies, where the key parameter was PDMS-gold controlled thickness. We resume the topic developed in this paper by the following points:

1) to provide the technological aspects and a basic physical description of the sensor;

2) to measure by means of an experimental setup suitable for robotic applications the sensitivity of the optimized gold/PDMS sensor prototype;

3) to finally provide a shape/roughness detection algorithm for robotic implementation.

\section{TeChnological Aspects AND the BASIC PHysical DESCRIPTION OF THE SENSOR BY an ANAlytical MODEL}

Fig. 1(a) illustrates the technology of the proposed optimized sensor prototype. The bottom half of the tapered fiber is embedded in the PDMS material in order to improve its mechanical stability.

The proposed prototype sensor is illustrated in Fig. 1(a) and (b). The tapered multimode $S_{i}$ fiber couples the EM field coming from a broad band lamp source with the flexible polymer-GNM.

The first prototype reported in [26] was based on a nonintegrated cap of the PDMS-Au material just placed on the tapered fiber. For this first prototype, we have observed lower sensitivity on the order of about $40 \mathrm{~g}$. The choice of the half-embedded fiber, as shown in Fig. 1, is due to the possibility of allowing an optical integrated system to provide better mechanical stability and finally perform higher sensitivity. An important point of optimization comes from an increase in the effective coupling interface between the tapered fiber and the GNM that increase pressure sensitivity.

The PDMS material is obtained by mixing a curing agent of a 1:10 weight ratio to a base monomer. We control the deposition of PDMS using the initial liquid state of PDMS; in particular, we added PDMS liquid droplets step by step in order to immerse only the bottom half of the tapered fiber, as illustrated in Fig. 1(a). About three days are necessary in order to reach the solid/elastomeric state of PDMS without heating. The prototype is obtained by depositing the GNM with a specific gold concentration.

As the generation of gold nanoparticles is controlled by chemical reduction of chloroauric acid salt, the key parameter to obtain a good dispersion is due to continuous stirring before the solidification process of PDMS.

The tapered fiber is fabricated by a coated silica/core silica multimode optical fiber (FG-365-LER Thorlabs fiber) by a controlled system. The system allows to pull uniformly the fiber by rotating it and simultaneously by burning the jacket and the cladding for some seconds (the number of seconds are related 
to the intensity and the distance of a flame) in order to obtain a $1 \mathrm{~cm}$ of the total tapered profile with about $5 \mathrm{~mm}$ of the central core region without the cladding. The whole dimension of the sensor in Fig. 1(b) is $3 \times 3 \mathrm{~cm}$ with $3 \mathrm{~mm}$ of PDMS. A part of PDMS is without gold [see Fig. 1(a)], which behaves as a mechanical elastomeric support (about $2 \mathrm{~mm}$ of thickness), and the remaining part is a sensitive part made of the tapered fiber embedded in the PDMS material with gold (about $1 \mathrm{~mm}$ of thickness). As shown in Fig. 1(a), the PDMS material with gold (i.e., the sensitive part) covers exactly the remaining diameter of the fiber by forming a meniscus. Previous studies demonstrate that the use of a PDMS polymer is able to generate gold nanoparticles by reducing gold precursors [26]-[28]. In this paper, we establish a very high gold concentration, which preserves the elastomeric properties of the GNM for realtime pressure detection (a time response of $0.6 \mathrm{~s}$ is experimentally checked by the DMA Q800 dynamical mechanical analyzer). In a specific case, we use chloroauric acid salt as the gold precursor in a water solution $\left(\mathrm{Mw}\left(\mathrm{HAuCl}_{4}\right)=\right.$ $339.785 \mathrm{~g} / \mathrm{mol}$ and $\left.\left[\mathrm{HAuCl}_{4}\right]=0.01 \mathrm{M}\right)$ with a concentration of about $10 \%$ in weight. The gold nanocomposite piece (i.e., the PDMS material with gold nanoparticles [26]-[28]) behaves as a cap and covers the remaining upper part of the tapered fiber. In this way, the contact interface becomes more efficient for light coupling between the tapered fiber and the GNM, particularly when pressure is applied. GNM deposition is controlled in order to cover perfectly the upper half of the tapered fiber, as indicated in the longitudinal section in Fig. 1(a). About four days are enough in order to transform the deposited liquid GNM into a solid elastomeric material. Due to the controlled GNMPDMS deposition, the contact surface increases the efficiency of the sensor because the whole tapered region is totally embedded in the GNM. The optimized total thickness of the GNM layer was found to be equal to half of the diameter of the fiber. The main physical effect of the sensor is due to the contact interface between the fiber and the GNM. After the application of pressure, the gold nanoparticles will approach the fiber surface (i.e., the contact interface between the tapered fiber and the GNM material), as indicated in Fig. 1(c). Near the contact surface, light is strongly scattered, as illustrated in Fig. 1(d), by increasing the intensity of light coupling [29] and, consecutively, by reducing the transmitted light intensity. The physical problem of the sensor can be explained by the following considerations. The broad lamp source excites the $\mathrm{LP}_{\mathrm{nm}}$ modes of the optical fiber solution of the wave equation [30] as follows:

$\left\{\frac{\partial^{2}}{\partial \rho^{2}}+\frac{1}{\rho} \frac{\partial}{\partial \rho}+\frac{1}{\rho^{2}} \frac{\partial^{2}}{\partial \phi^{2}}+\varepsilon_{r} K_{0}^{2}-\beta^{2}\right\} \psi_{\mathrm{nm}}(\rho, \vartheta)=0$

where $\rho, \theta$, and $\phi$ represent the cylindrical coordinates, and $\psi_{\mathrm{nm}}$ is the function representing the optical fiber modes.

The eigenvalue equation (i.e., a characteristic equation) provides the propagation constants of the $\mathrm{LP}_{\mathrm{nm}}$ mode. It is derived by matching the field components at the core-cladding interface given as [28]

$$
\kappa \cdot \frac{J_{\nu-1}(\kappa a)}{J_{\nu}(\kappa a)}=i \gamma \cdot \frac{K_{\nu-1}(i \gamma a)}{K_{\nu}(i \gamma a)}
$$

where $a$ is the radius of the core, and $\kappa$ and $\gamma$ are the transverse propagation constants defined as

$$
\begin{aligned}
& \kappa=\sqrt{n_{c}^{2} k_{0}^{2}-\beta^{2}} \\
& \gamma=\sqrt{\beta^{2}-n_{g}^{2} k_{0}^{2}}
\end{aligned}
$$

where $k_{0}$ is the free-space wavenumber, $\beta$ is the longitudinal propagation constant, and $n_{c}$ and $n_{g}$ are the core and cladding refractive indexes, respectively. In order to evaluate the number of propagating $\mathrm{LP}_{\mathrm{nm}}$ modes and the relative propagation constants, it is useful to define the dimensional parameter as

$$
V=\frac{2 \pi a}{\lambda} \sqrt{n_{c}^{2}-n_{g}^{2}}=\sqrt{U^{2}+W^{2}}
$$

where $U=U_{\mathrm{nm}}=\kappa_{\mathrm{nm}} a$, and $W=W_{\mathrm{nm}}=\gamma_{\mathrm{nm}} a$.

Due to the tapered profile, the fiber modes $\Psi_{n m}$ calculated by (1)-(4) will couple with the PDMS-Au cladding material. As shown in Fig. 2(a), the tapered profile of the multimodal optical fiber defines the effective permittivity $\varepsilon_{\mathrm{ei}}$ for each section of the profile and, consecutively, defines step discontinuity able to couple the light with the PDMS/PDMS-Au claddings. When pressure force is applied, the $n_{g 2}$ index will increase by moving along the direction indicated by the top red arrow in Fig. 2(b). As shown in Fig. 1(c) and (d), more gold micro/nanoparticles will move on the fiber interface by changing the refractive index of the PDMS-Au (we assume a high gold concentration for the region with higher permittivity in contact with the tapered region and a reduction of $n_{g 2}$ of a different extent). The electric field radiated by the tapered profile can be expressed as superposition of plane waves as [30]

$$
\begin{aligned}
E^{r}(r, \theta, \varphi) \approx j k_{0} \cdot & \frac{e^{-j\left[k_{0} r+\Delta \phi\right]}}{2 \pi r} \\
& \cdot\left[\cos \theta \cdot \sum_{n m} A^{n m}(\theta, \varphi) \cdot e^{j \beta_{n m} z}\right]
\end{aligned}
$$

where $\Delta \phi$ represents the phase-correction contribution function of the slanted angle of the tapered profile. The modes of the tapered fiber will exchange power with the PDMS-Au cladding by defining the coupling coefficient as follows [31]:

$$
C_{\psi_{n m}, E^{r}}(z)=\omega \int_{-\infty}^{\infty} \int_{-\infty}^{\infty} \Delta \varepsilon_{e}(z) \psi_{n m} E^{r} d x d y
$$

where $\Delta \varepsilon_{e}$ indicates the variation of the effective permittivity along the $z$-longitudinal direction. The coupling coefficient $C$ includes the information of the reduction of transmitted light at the output of the fiber. Being the $C$ function of the variation of the effective permittivity, major EM coupling (and, consecutively, a major reduction of transmitted light intensity) will happen corresponding to a strong variation of the effective permittivity. This strong variation is obtained when a pressure is applied [see Fig. 2(b)]. 


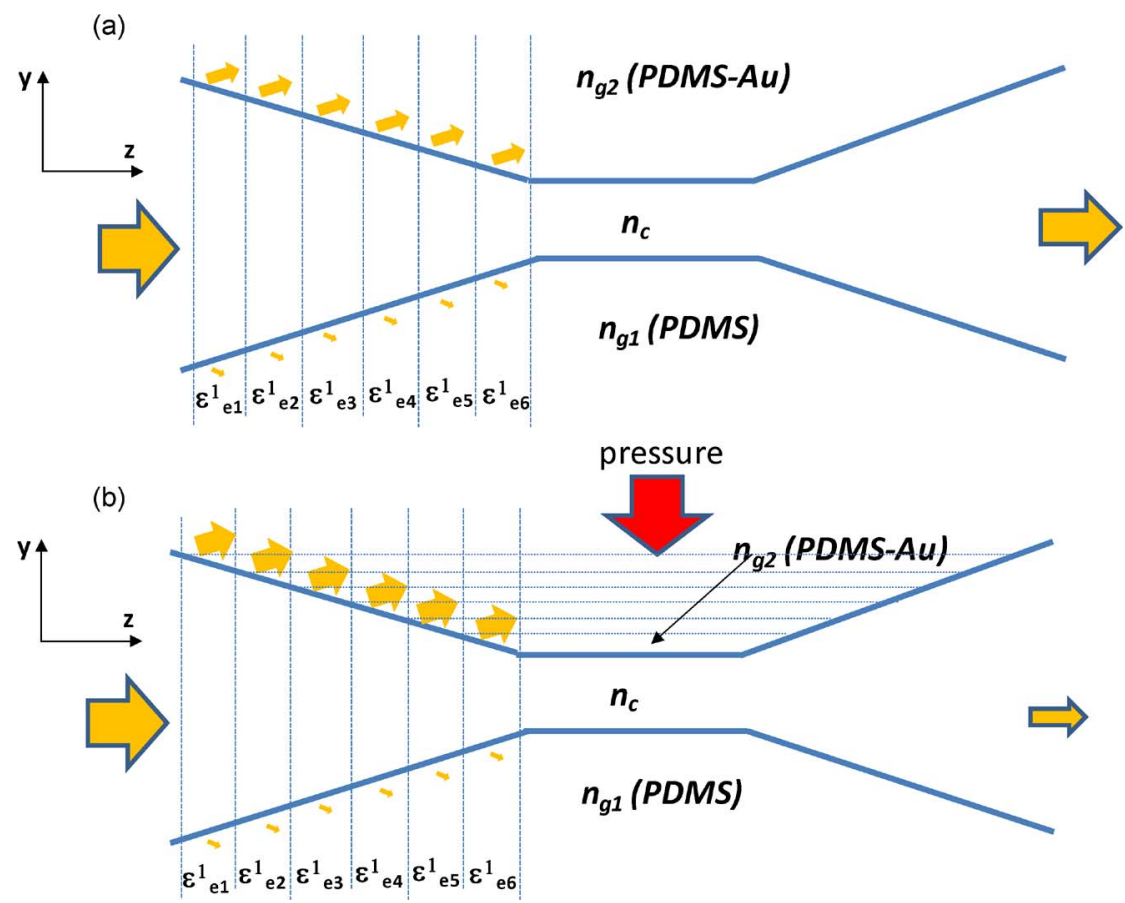

Fig. 2. (a) Light coupling by means of dielectric discontinuity due to the tapered profile (without applied pressure forces, the PDMS-Au cladding is uniform). (b) Light coupling between the nonuniform PDMS-Au cladding and the optical fiber.

(a)

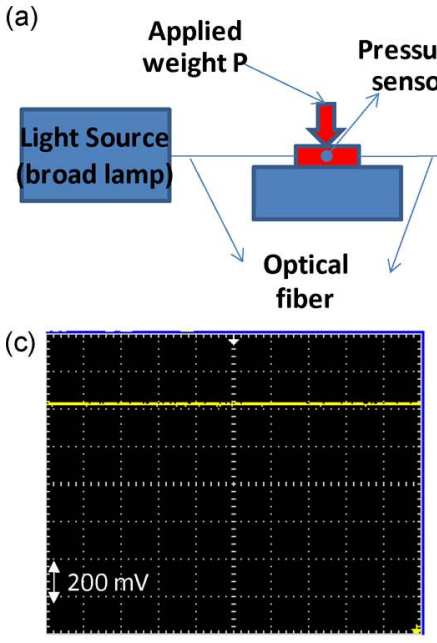

(f)

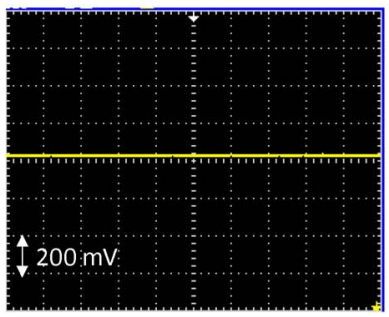

Photo
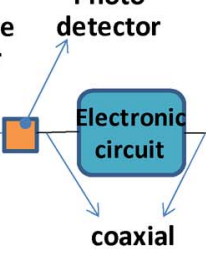

cables

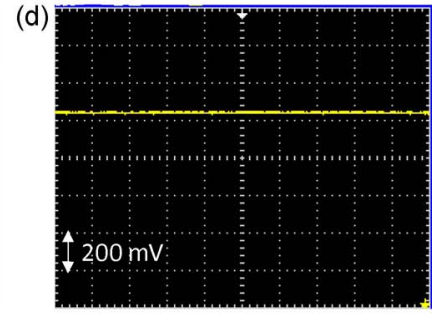

(g)

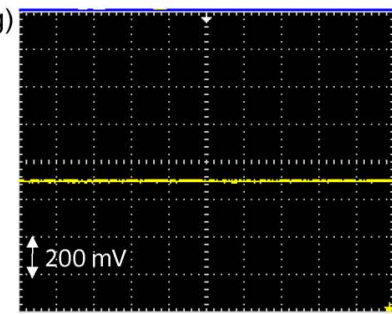

(b)

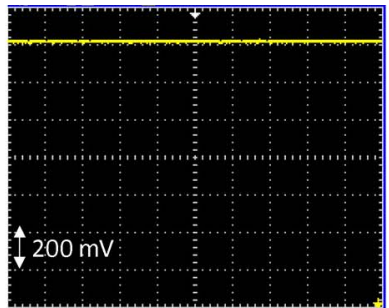

(e)

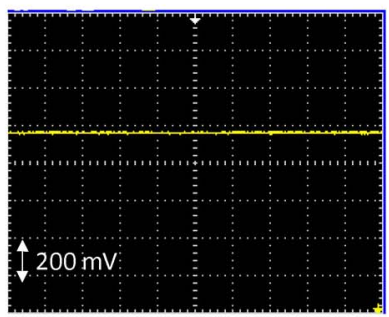

(h)

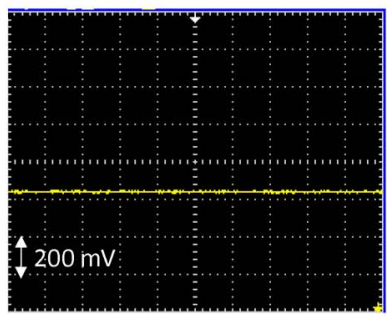

Fig. 3. (a) Schematic diagram of the experimental setup suitable for robotic finger implementation. (b) Electric signal detected by switching on the broad lamp and without applying pressure forces. (c) Electric signal detected by applying (c) $P=5 \mathrm{~g}$, (d) $P=10 \mathrm{~g}$, (e) $P=15 \mathrm{~g}$, (f) $P=20 \mathrm{~g}$, (g) $P=30 \mathrm{~g}$, and (h) $P=40 \mathrm{~g}$ as weight. Each square unit represents $200 \mathrm{mV}$.

\section{Electrical Measurement Procedures}

Electrical measurement procedures are performed using the experimental setup in Fig. 3(a). An optical broad lamp (800$1500 \mathrm{~nm}$ ) is connected with the tapered fiber sensor. The output of the fiber sensor is connected with a Thorlabs DET 210 high- speed silicon photodetector in order to convert an optical signal into an electrical one. For its small dimensions, this detector is suitable for integration in a robotic arm. A pressure sensor sample is fixed on a system, which assures mechanical stability during the application of the pressure forces. By applying 

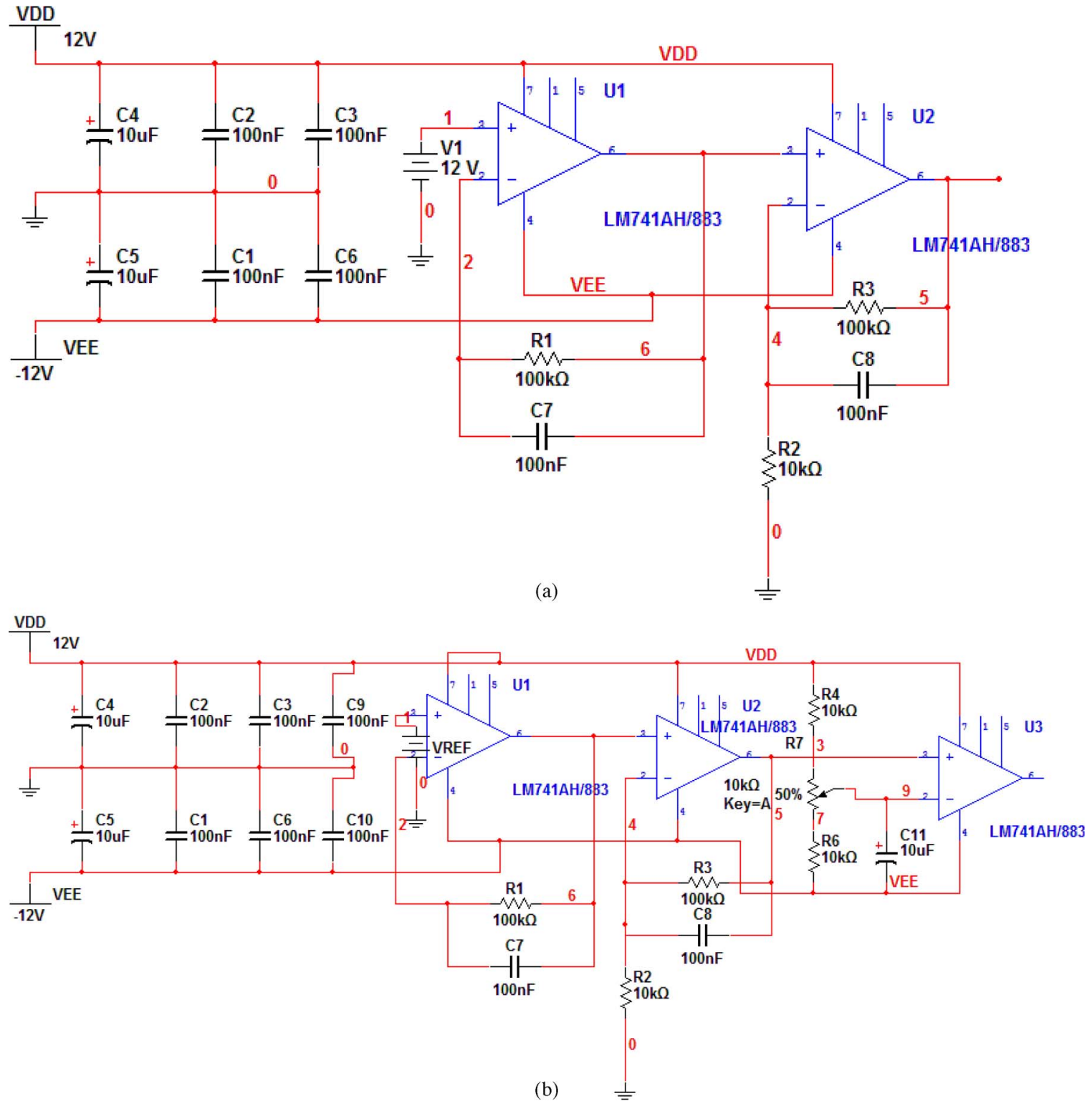

Fig. 4. (a). Double-stage circuit for sensor characterization. (b) Double-stage circuit for sensor characterization with a threshold comparator.

different weights step by step to the sensors, a significant variation of the transmitted light intensity occurs also for a few grams, as indicated in Fig. 3(b)-(h). The reading of an electrical signal is performed by one of the electrical circuits, as reported in Fig. 4(a) and (b), which is connected to an electronic oscilloscope by a coaxial cable. The coaxial cable is also used for the connection between the photodiode and the electronic circuit. The double stage using $741 \mu \mathrm{A}$ is necessary to amplify the signal coming from the pressure sensor to be displayed due to a digital scope [see Fig. 4(a)]. In order to control the output in function of the input and since the signal coming from the pressure sensor is almost weak, a specific circuit using a threshold comparator is included at the end of the two stages according to Fig. 4(b). The comparator switches from 1/3 to 2/3 V; the switching, concerning the output of the second operational amplifier, is performed by means of a trimmer. The threshold comparison is necessary because it allows a correct regulation of the voltage because, during the use of different weights, sudden variations of voltage appear, since the top part of the sensor is similar to a spring, and must be compensated by acting on the trimmer, hence, on the input voltage upstream of the comparator.

The proposed sensor exhibits high reactivity of about $0.6 \mathrm{~s}$. The electrical/mechanical time response of the sensor is due to the elastomeric properties of the GNM material.

During the characterization of the prototype, we used a controlled system of weights, which did not move the sensor during the experimental process, and allowed to increase the pressure in steps applied using a few grams.

In Figs. 5 and 6, we show the first three series of measurements and the average value of five series of measurements using the experimental setup in Fig. 3, respectively. In particular, in Fig. 5, we illustrate three series of measurements, and each point of one series is obtained by adding step-by-step calibrated weights of 5 and $10 \mathrm{~g}$ on the sample. For each measurement (point of the series), a stable voltage is checked (no oscillations 


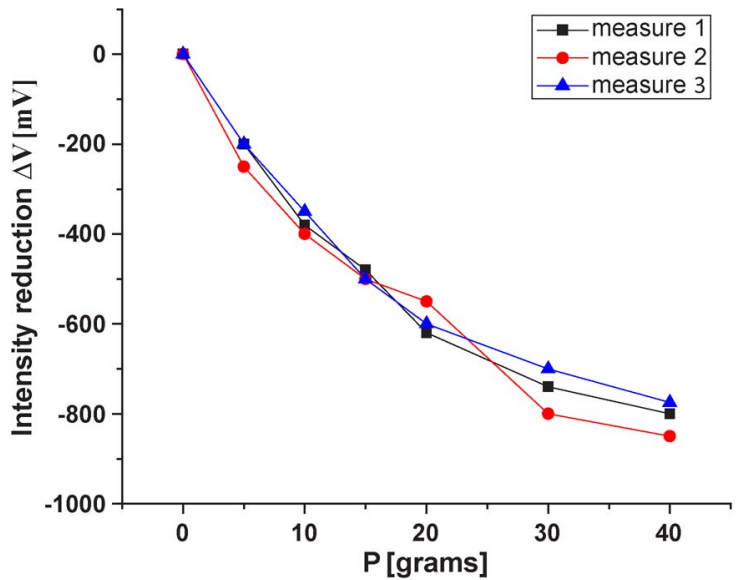

Fig. 5. First three measurements of the pressure sensor prototype by means of the experimental setup reported in Fig. 1.

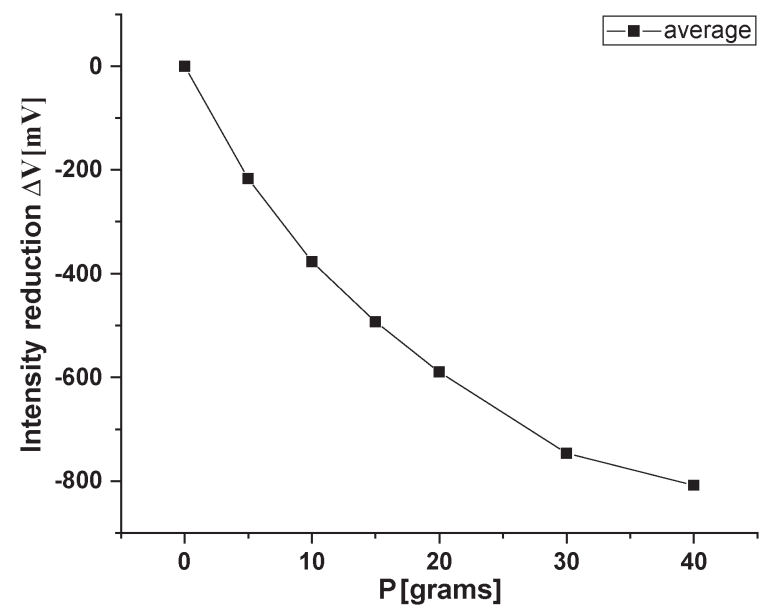

Fig. 6. Plot of the average of five measurements. The plot indicates the sensitivity of the sensor.

are observed). We observe from the final trend in Fig. 6 that the sensitivity of the sensor decreases by increasing the applied weights: This is due to the mechanical properties of the GNM material and can change with gold concentration. We have applied the weights exactly on the axis of the tapered fiber by using an $(x, y)$ micrometric table system. Sensitivity on the order of $5 \mathrm{~g}$ is found.

The high sensitivity of the proposed sensor allows utilization in an accurate robotic system.

\section{Application: Tactile Sensing for Robotic Systems AND THE Algorithm of Shape Detection}

The proposed sensor can be easily implemented in robotic tactile systems as a finger of a robotic hand. Using small laser diode sources and small photodiode detectors, the sensitive pressure sensor requires small spaces and assures a very fast time response of detection. In addition, as technologically proven [see the inset in Fig. 7(a)], the tapered fiber sensor can be bended on a support by allowing to consider the application in Fig. 7(a) representing the detection of a bidimensional geometrical profile.
The high sensitivity of a few grams, checked by the measurements reported in the previous section, allows to design an algorithm for the detection of shapes or roughness based on the concept of the equivalent applied weights. The equivalent applied weight $P_{\text {ei }}$ can be estimated by considering the sensitivity response in Fig. 6. By applying the displacement multiple of $\Delta y$ to the roughness profile in Fig. 7(a), the sensor will respond by defining the shape. We explain in details the principle of detection by the example in Fig. 7(b) reported by the following steps (we report as an example only the first steps in order to understand the detection procedure) and by the algorithm in Fig. 7.

1) The system is calibrated by setting $P_{e 0}$ obtained from a low pressure (equivalent for the example to an applied weight of $P_{e 0}=5 \mathrm{~g}$ ) at position $y=0$.

2) The sensor is moved up by $-\Delta y$, to the right by $+\Delta x$, and down by $+\Delta y$. After $+\Delta y$, no pressure force is detected, and consecutively, the sensor is moved down again from $+\Delta y$ by detecting an equivalent pressure of $P_{e 1}>P_{e 0}$. The coordinate of the profile in point 1 is $y_{1}=(-\Delta y+\Delta y+\Delta y)-\Delta h_{1}\left(P_{e 1}\right)$.

3) The sensor is moved up by $-\Delta y$, to the right by $+\Delta x$, and down by $+\Delta y$. After $+\Delta y$, no pressure force is detected, and consecutively, the sensor is moved down again from $+\Delta y$ by detecting an equivalent pressure of $P_{e 2}>P_{e 1}$. The coordinate of the profile in point 2 is $y_{2}=y_{1}+\Delta y-\Delta h_{2}\left(P_{e 2}\right)$ in opposition to $\Delta h_{2}\left(P_{e 2}\right)>$ $\Delta h_{1}\left(P_{e 1}\right)$.

4) The sensor is moved up by $-\Delta y$, to the right by $+\Delta x$, and down by $+\Delta y$. After $+\Delta y$, no pressure force is detected, and consecutively, the sensor is moved down again from $+\Delta y$ by detecting an equivalent pressure of $P_{e 3} \cong P_{e 0}$. The coordinate of the profile in point 3 is $y_{3}=y_{2}-\Delta h_{3}\left(P_{e 3}\right)$ in opposition to $\Delta h_{3}\left(P_{e 3}\right)=0$.

5) The sensor is moved up by $-\Delta y$, to the right by $+\Delta x$, and down by $+\Delta y$. After $+\Delta y$, no pressure force is detected, and consecutively, the sensor is moved down again from $+\Delta y$ three times by detecting an equivalent pressure of $P_{e 4} \cong P_{e 0}$. The coordinate of the profile in point 4 is $y_{4}=y_{3}+3 \Delta y-\Delta h_{4}\left(P_{e 4}\right)$ in opposition to $\Delta h_{4}\left(P_{e 4}\right)=0$.

The number of the steps of the procedure will be defined in the logic system by means of a stop condition (see Fig. 8) defined by the user. We observe that, as also reported in the algorithm in Fig. 8, that step $\Delta x$ can be furthermore decreased, in a feedback system, in order to increase spatial resolution. The signals coming from each sensitive sensors can be detected by photodiode detectors and can be processed by a logic network, providing, by a controlled feedback system, commands about the possible movements of the sensors also in the 3-D space. Spatial resolution can be increased also by reducing the size of the sensor (e.g., simply by fabricating a thin system). The limit of resolution is defined by the diameter of the fiber.

\section{CONCLUSiOn}

In this paper, we have presented a new concept of an innovative optical tactile sensor based on PDMS-GNMs and its 


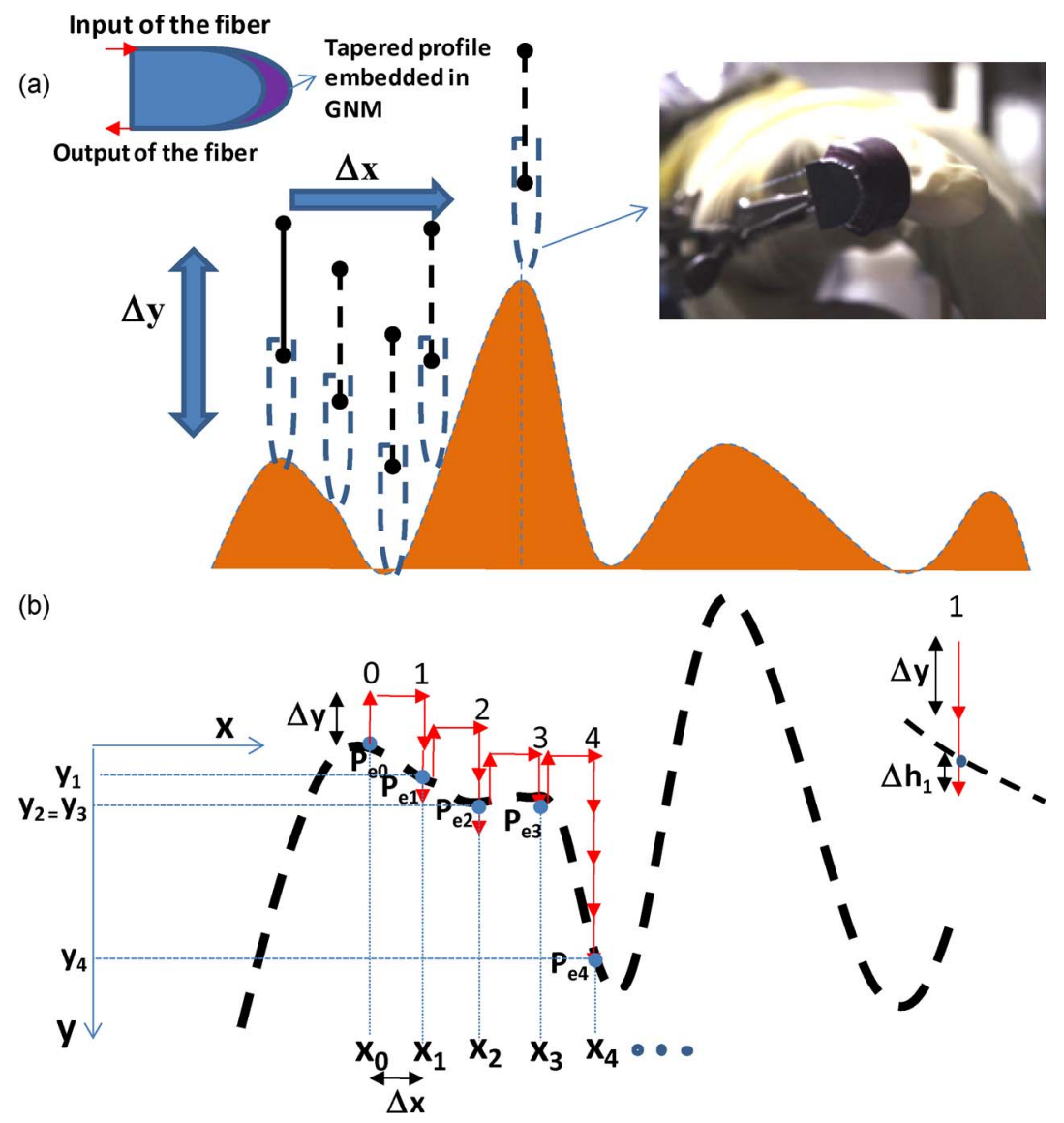

Fig. 7. (a) Schematic diagram of the robotic finger as an application of shape/roughness profile detection. The left part of the inset shows the schematic plot of the implemented robotic finger. The right part of the inset shows the tactile robotic finger prototype where the tapered fiber inside a polyvinyl chloride (PVC) support representing the finger shape and improving mechanical stability; the tapered profile is glued on the PVC support (the PVC support is embedded in the GNM) by means of Thorlabs epoxy glue for fiber optic connectors. (b) Example of profile detection. The inset shows the zoomed sketch of step 1.

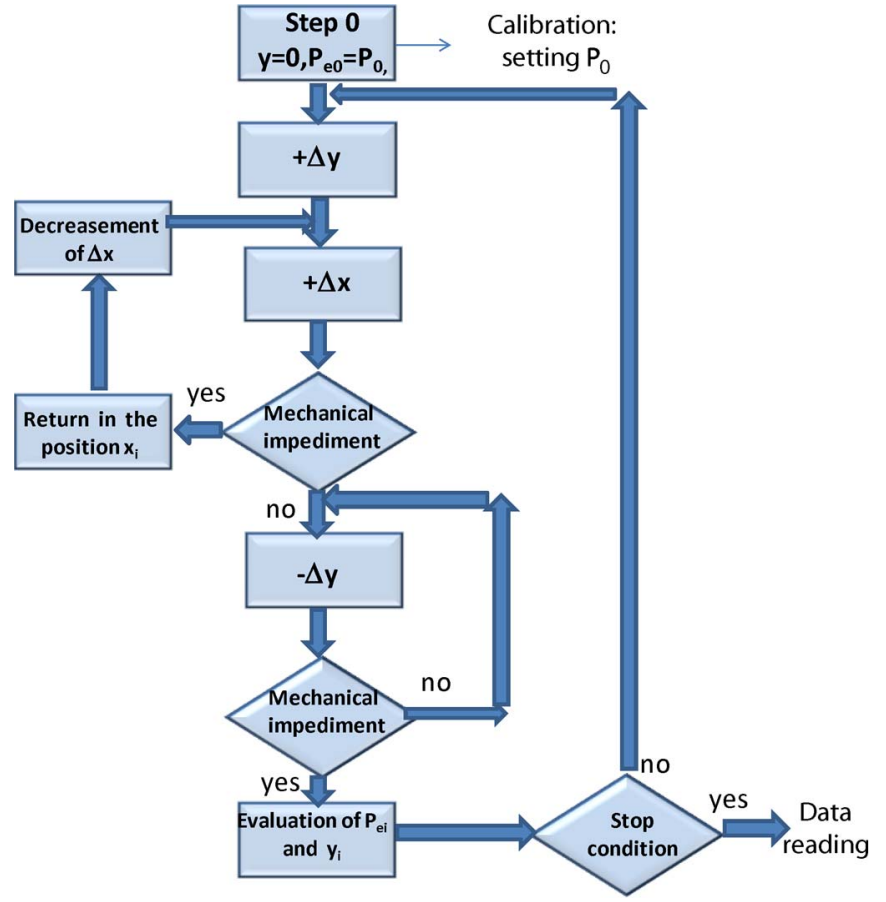

Fig. 8. Generic algorithm for roughness/shape detection in an intelligent robotic system. electronic characterization. In particular, in this paper, we have proposed a high-sensitivity sensor, which allows to measure pressure forces corresponding to weights of $5 \mathrm{~g}$ and can be easily implemented in tactile robotic systems including shape and dielectric permittivity sensing. An example of an algorithm regarding bidimensional roughness/shape detection is presented. The novelties of this paper are in a new approach of tactilesensing measurements based on optical coupling of GNMs. A lot of studies on tactile sensing is based on the capacitive and/or piezoresistive effect; the proposed original optical sensor reproduces the pressure effect as a reduction of the transmitted optical intensity. This choice is due to the well-known velocity of light in optical waveguides by increasing the reactivity of the sensor. The sensor is highly responsive for light coupling efficiency due to light scattering of micro/nanoparticles and for the good elastomeric properties of the PDMS material. High sensor reactivity allows to obtain sensitivity on the order of $5 \mathrm{~g}$. The basic physical principle of this new approach of measurements is mainly in light coupling in new nanocomposite materials. In the literature, there are no sensors fabricated by the approach of this paper. The implementation of the final sensor as a robotic finger is designed in order to integrate supplementary sensors such as ultrasonic sensing for approaching distance control. The proposed sensor is currently suitable for soft pressure of a few grams. The next pressure sensor generation 
will consider the use of higher pressure by taking into account different PDMS-curing agent concentrations and designed rigid supports. Our goal is to obtain pressure sensors adaptable at different pressure magnitude values by acting on the chemical elastomeric rigidity of PDMS. The mechanical control system and signal processing are currently under investigation as further work.

\section{ACKNOWLEDGMENT}

The authors would like to thank A. Masciullo and D. Mangiullo for the technical support.

\section{REFERENCES}

[1] S. M. Firdaus, I. A. Azid, O. Sidek, K. Ibrahim, and M. Hussien, "Enhancing the resistivity of a mass-based piezoresistive micro-electromechanical systems cantilever sensor," IET Micro Nano Lett., vol. 5, no. 2, pp. 85-90, Apr. 2010.

[2] B. J. Kane, M. R. Cutkosaky, and T. A. Kovacs, "A traction stress sensor array for use in high resolution robotic tactile image," J. Microelectromech. Syst., vol. 9, no. 4, pp. 425-434, 2000.

[3] D. J. Beebe, A. S. Hseih, D. D. Denton, and R. G. Radwin, "A silicon force sensor for robotics and medicine," Sens. Actuators A, Phys., vol. 50, no. $1 / 2$, pp. 55-65, Aug. 1995.

[4] J. I. Lee, X. Huang, and P. B. Chu, "Nanoprecision MEMS capacitive sensor for linear and rotational positioning," IEEE J. Microelectromech. Syst., vol. 18, no. 3, pp. 660-670, Jun. 2009.

[5] B. L. Gray and R. S. Fearing, "A surface micromachined micro tactile sensor array," in Proc. IEEE Int. Conf. Robot. Autom., 1996, pp. 1-6.

[6] M. Leineweber, G. Pelz, M. Schmoidt, H. Kappert, and G. Zimmer, "New tactile sensor chip with silicone rubber cover," Sens. Actuators A, Phys., vol. 84, no. 3, pp. 236-245, Sep. 2000.

[7] C. Li, P. M. Wu, L. A. Shutter, and R. K. Narayan, "Dual-mode operation of flexible piezoelectric polymer diaphragm for intracranial pressure measurement," Appl. Phys. Lett., vol. 96, no. 5, pp. 053502-053502-3, Feb. 2010.

[8] E. S. Kolesar and C. S. Dyson, "Object image with a piezoelectric robotic tactile sensor," J. Microelectromech. Syst., vol. 4, no. 2, pp. 87-96, Jun. 1995.

[9] R. R. Reston and E. S. Kolesar, "Robotic tactile sensor array fabricated froma piezoelectric polyvinylidine fluoride film," in Proc. IEEE NAECON, 1990, pp. 1139-1144.

[10] J. Engel, J. Chen, Z. Fan, and C. Liu, "Polymer micromachined multimodal tactile sensors," Sens. Actuators A, Phys., vol. 117, no. 1, pp. 50-61, Jan. 2005.

[11] J. L. Schneiter and T. B. Sheridan, "Robotics and Computer-Integrated Manufacturing," Comput. Integr. Manuf. Syst., vol. 1, no. 1, pp. 65-71, 1984.

[12] H. S. Ko, C. W. Liu, and C. Gau, "Novel fabrication of a pressure sensor with polymer material and evaluation of its performance," J. Micromech. Microeng., vol. 17, no. 8, pp. 1640-1648, Aug. 2007.

[13] J. Martin, W. Bacher, O. F. Hagena, and W. K. Schomburg, "Strain gauge pressure and volume-flow transducers made by thermoplastic molding and membrane transfer," in Proc. MEMS, 1998, pp. 361-366.

[14] A. V. Shirinov and W. K. Schomburg, "Polymer pressure sensor from PVDF," in Proc. 20th Eurosensors, 2006, vol. 1, pp. 84-85.

[15] K. Arshak, D. Morris, A. Arshak, O. Korostysnska, E. Jafer, D. Waldron, and J. Harris, "Development of polymer-based sensors for integration into a wireless data acquisition system suitable for monitoring environmental and physiological processes," Biomol. Eng., vol. 23, no. 5, pp. 253-257, Oct. 2006

[16] H. S. Ko and C. Gau, "Bonding of a complicated polymer microchannel system for study of pressurized liquid flow characteristics with the electric double effect," J. Micromech. Microeng., vol. 19, no. 11, pp. 1-13, Nov. 2009.

[17] T. Shinohara, S. Dohta, and H. Matsushita, "Development of a soft actuator with a built-in flexible displacement sensor," in Proc. 9th Int. Conf. New Actuator, 2004, pp. 383-386.

[18] K. Kanamori, "Electroconductive rubber with corbonblack, (4) Developments of electro-conductive rubber and applied devices," J. SRIJ, vol. 58, no. 9, pp. 597-603, 1985.
[19] K. Kure, T. Kanda, K. Suzumori, and S. Wakimoto, "Intelligent FMA using flexible displacement sensor with paste injection," in Proc. IEEE Int. Conf. Robot. Autom., 2006, pp. 1012-1017.

[20] R. Tajima, S. Kagami, M. Inaba, and H. Inoue, "Development of soft and distributed tactile sensors and the application to a humanoid robot," Adv. Robot., vol. 16, no. 4, pp. 381-397, 2002.

[21] T. Kanda, H. Ishiguro, T. Ono, M. Imai, and R. Nakatsu, "Development and evaluation of an interactive humanoid robot 'Robovie'," in Proc. ICRA, 2002, pp. 4166-4173.

[22] S. Omata, Y. Murayama, and C. E. Constantinou, "Real time robotic tactile sensor system for the determination of the physical properties of biomaterials," Sens. Actuators A, Phys., vol. 112, no. 2/3, pp. 278-285, May 2004.

[23] S. Sokhanvar, M. Packirisamy, and J. Dargahi, "A multifunctional PVDFbased tactile sensor for minimally invasive surgery," Smart Mater. Struct. vol. 16, no. 4, pp. 989-998, Aug. 2007

[24] A. P. Miller, W. J. Peine, J. S. Son, and Z. T. Hammoud, "Tactile imaging system for localizing lung nodules during video assisted thoracoscopic surgery," in Proc. IEEE Int. Conf. Robot. Autom., Roma, Italy, 2007, pp. 2996-3001.

[25] J. S. Heo, J. H. Chung, and J. J. Lee, "Tactile sensor array using fiber Bragg grating sensors," Sens. Actuators A, Phys., vol. 126, no. 2, pp. 312-327, Feb. 2006.

[26] Q. Zhang, J. J. Xu, Y. Liu, and H. Y. Chen, "In-situ synthesis of poly(dimethylsiloxane)- gold nanoparticles composite films and its application in microfluidic system," Lab chip, vol. 8, no. 2, pp. 352-357, 2008.

[27] C. E. Hoppe, C. Ridriguez-Abreu, M. Lazzari, M. A. Lopez-Quintela, and C. Solans, "One-pot preparation of gold-elastomer nanocomposites using PDMS- graft - PEO copolymer micelles as nanoreactors," Phys. Stat. Sol. (A), vol. 205, no. 6, pp. 1455-1459, Jun. 2008.

[28] A. Goyal, A. Kumar, P. K. Patra, S. Mahendra, S. Tabatabaei, P. J. J. Alvarez, G. Jhon, and P. M. Ajayan, "In situ synthesis of metal nanoparticles embedded free standing multifunctional PDMS films," Macromol. Rapid Commun., vol. 30, pp. 1116-1122, 2009.

[29] A. Massaro, F. Spano, P. Cazzato, R. Cingolani, and A. Athanassiou, "Real time optical pressure sensing for tactile detection using gold nanocomposite material," in Proc. MNE, P NANO, 2010, p. 12.

[30] A. Massaro, L. Pierantoni, and T. Rozzi, "Far-field radiation of optical fibers with tapered end," IEEE J. Light. Technol., vol. 24, no. 8, pp. 3162-3168, Aug. 2006.

[31] A. Massaro, L. Pierantoni, and T. Rozzi, "Development of the EM coupling in laminated multilayered 3D optical waveguides," Int. J. Num. Model., vol. 18, no. 3, pp. 237-253, May 2005.

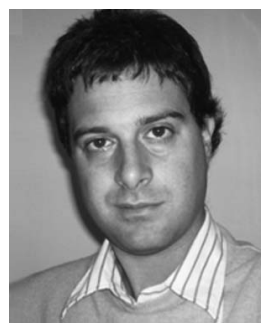

Alessandro Massaro received the Laurea degree in electronic engineering and the Ph.D. degree in telecommunication engineering from Marche Polytechnic University, Ancona, Italy, in 2001 and 2004, respectively.

From 2004 to 2006, he worked as a Postdoctoral Research Scientist with the Department of Electromagnetism and Bioengineering, Marche Polytechnic University. In 2006, he spent two years in research and development with the medical and industrial optics industry (for endoscope design and optical systems). For two years, He was with the National Nanotechnology Laboratory, National Research Council-National Institute for the Physics of Matter, Lecce, Italy, as a Principal Investigator. Currently, He is the Team Leader of the Robotics Laboratory 1, Center for Biomolecular Nanotechnologies, Italian Institute of Technology, Lecce. His research interests include design and modeling of photonic band-gap circuits, development of computer-aided design tools in the area of integrated optics, microelectromechanical/nanoelectromechanical system technology and systems, and smart material design.

Dr. Massaro is a member of the European Microwave Association. 


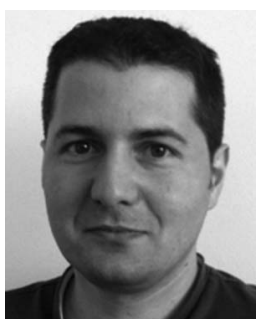

Fabrizio Spano was born in Mulhouse, France, in April 1972. He received the Ph.D. degree in chemistry, financed by Messier-Bugatti [formerly named as the National Company for the Design and Construction of Aviation Engines (SNECMA)], from the Institute of Chemistry of Surfaces and Interfaces (ICSI-CNRS), Mulhouse, by submitting a thesis entitled Topographies of Rubbing Surfaces of Carbon/Carbon Composite Materials, Formation Mechanisms of Third Body and Effects on Friction and Wear.

During his doctoral years, he deals with tribology, rheology, composite materials, granular mechanics, and micro/nanoparticles. In 2004, he worked as a Postdoctoral Research Scientist with the Institute for Macromolecular Studies-National Research Council (ISMAC-CNR), Milan, Italy, in the framework of the research training networking Nanochannel project where he worked on micro/nanoorganization of materials for various applications (e.g., optics, energy transfer, light harvesting, etc.). From 2006 to 2009, he spent three years with the Biomicroelectromechanical Sector, Nanotechnology and Life Science Division, Swiss Center for Electronics and Microtechnology (CSEM SA), Neuchâtel, Switzerland, as an R\&D Engineer, where he dealt with tissue engineering, 3-D scaffolds, nerve regeneration, electrospun materials, and biotechnology in general. Since November 2009, he has been with the Smart Materials Division, Center for Biomolecular Nanotechnologies, Italian Institute of Technology, Lecce, Italy, and dealt with nanocomposite materials for various applications and, in particular, with gold nanocomposite materials obtained by laser texturing.

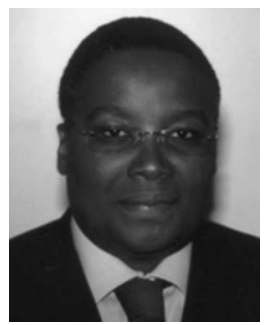

Aimé Lay-Ekuakille received the M.S. and Ph.D. degrees in electronic engineering, the M.S. degree in clinical engineering, and the Ph.D. degree in environmental impact assessment from the University of Salento, Leece, Italy.

From 1993 up to 2001, he was an Adjunct Professor of measurements and control systems in different universities. In September 2000, he was with the Measurement and Instrumentation Group, Department of Innovation Engineering, University of Salento. He was the Director of a health and environment municipal department and of different private companies in the field of industrial plants, environmental measurements, and nuclear and biomedical measurements. He was a Technical Advisor of the Italian government for highrisk plants. Since 2003, he has become the Leader of that scientific group and, currently, the Coordinator of the Measurement and Instrumentation Laboratory, University of Salento. He also teaches electronic instrumentation for measurements and reliability for electronic materials and devices. His main research interests include environmental, industrial, and biomedical instrumentation and measurements.

Dr. Lay-Ekuakille was the Chair of the IEEE-sponsored circuits and instrumentation systems/Systems Signal Devices Conference and the Co-Chair of the 2010 International Conference on Software Testing, Verification and Validation. $\mathrm{He}$ is a member of the TRANSACTIONS ON Systems Signal Devices editorial board. He is the Guest Editor of the IEEE Sensors Journal and the Associate Editor of the International Journal on Smart Sensing and Intelligent Systems and other international journals. He is the Founder and the Editor-in-Chief of International Journal of Measurement Technologies and Instrumentation Engineering published by the US IGI Global, Hershey, PA.

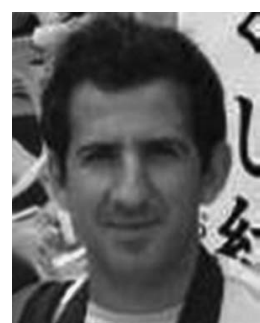

Italy, in 2008.

Since 2001, He has been with the National Nanotechnology Laboratory, Institute of Nanoscience, National Research Council, Lecce, as an Electronic Technician of the spectroscopy laboratory. His research interests include vacuum systems, cryogenic systems, electronic apparatus, lasers and their maintenance, photo luminescence setups in visible and infrared ranges, molecular beam epitaxy, and sputtering systems assistance.

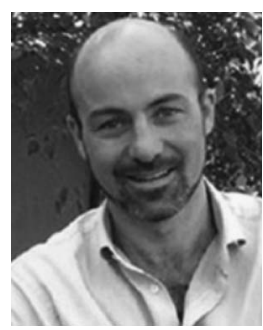

Roberto Cingolani was born in Milan, Italy, on December 1961. He received the Ph.D. degree in physics from Scuola Normale Superiore di Pisa, Pisa, Italy.

From 1989 to 1991, he was a Staff Member with Max Planck Institute for Solid State Research, Stuttgart, Germany. In 1997, he was a Visiting Scientist with Tokyo University, Tokyo, Japan. Since 2000, he has been a Full Professor of experimental physics with the University of Salento, Lecce, Italy. In 2004, he was the Scientific Director of the Italian Institute of Technology, Genova, Italy. He is the author or coauthor of more than 500 papers in international journals. He is the holder of 20 patents. His research interests include nanotechnology and nanofabrication, semiconductor physics and devices, molecular organic nanostructures and devices, and bionanotechnology.

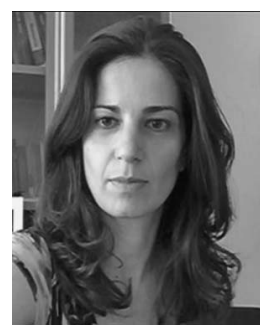

Athanassia Athanassiou Received the B.S. degree in physics from the University of Ioannina, Ioannina, Greece, in 1996; the M.Sc. degree from the Department of Physics, University of Manchester, Manchester, U.K., in 1997; and the Ph.D. degree from the Department of Physics, University of Salford, Salford, in 2000

From 2000 to 2005, she was a Researcher with the Foundation for Research and Technology-Hellas (FORTH) in Heraklion, Crete, Greece. From September 2003 to September 2005, she was with the Technological University of Crete, School of Applied Technology, Chania, Greece, as an Academic Staff. Since January 2006, she has been a Senior Researcher with the Nanocomposite and Responsive Materials Division, National Nanotechnology Laboratory, Institute of Nanotechnology, National Research Council, Lecce, Italy. In June 2006 and for two years, she was with the Italian Institute of Technology, Genova, Italy, as a Scientist responsible for the development and initiation of the optical spectroscopy-microscopy and Material Science Laboratory-Facility that at that moment is part of the nanophysics unit. Since September 2009, she has been a Coordinator of the smart material platform with the Center for Biomolecular Nanotechnologies, Italian Institute of Technology, Lecce. She is the author of 50 articles in refereed journals, the author or coauthor of several book chapters, and has several oral and invited contributions to international conferences. Her research interests include fabrication, development, processing, and characterization of nanocomposite materials; smart materials responsive to external stimuli; steady-state/transient spectroscopy; laser-matter interactions; and surface science. 